\title{
Unravelling the genes forming the wing pattern supergene in the polymorphic butterfly Heliconius numata
}

Suzanne V. Saenko ${ }^{1 \dagger}$, Mathieu Chouteau ${ }^{2 \dagger}$, Florence Piron-Prunier ${ }^{1}$, Corinne Blugeon ${ }^{3}$, Mathieu Joron ${ }^{4}$ and Violaine Llaurens ${ }^{1 *}$ (1)

\begin{abstract}
Background: Unravelling the genetic basis of polymorphic characters is central to our understanding of the origins and diversification of living organisms. Recently, supergenes have been implicated in a wide range of complex polymorphisms, from adaptive colouration in butterflies and fish to reproductive strategies in birds and plants. The concept of a supergene is now a hot topic in biology, and identification of its functional elements is needed to shed light on the evolution of highly divergent adaptive traits. Here, we apply different gene expression analyses to study the supergene $P$ that controls polymorphism of mimetic wing colour patterns in the neotropical butterfly Heliconius numata.
\end{abstract}

Results: We performed de novo transcriptome assembly and differential expression analyses using high-throughput Illumina RNA sequencing on developing wing discs of different H. numata morphs. Within the $P$ interval, 30 and 17 of the 191 transcripts were expressed differentially in prepupae and day-1 pupae, respectively. Among these is the gene cortex, known to play a role in wing pattern formation in Heliconius and other Lepidoptera. Our in situ hybridization experiments confirmed the relationship between cortex expression and adult wing patterns.

Conclusions: This study found the majority of genes in the $P$ interval to be expressed in the developing wing discs during the critical stages of colour pattern formation, and detect drastic changes in expression patterns in multiple genes associated with structural variants. The patterns of expression of cortex only partially recapitulate the variation in adult phenotype, suggesting that the remaining phenotypic variation could be controlled by other genes within the $P$ interval. Although functional studies on cortex are now needed to determine its exact developmental role, our results are in accordance with the classical supergene hypothesis, whereby several genes inherited together due to tight linkage control a major developmental switch.

Keywords: Supergene, Wing pattern, Lepidoptera, Heliconius butterflies, Transcriptome, RNA sequencing, Gene expression

*Correspondence: Violaine.Llaurens@mnhn.f

tSuzanne V. Saenko and Mathieu Chouteau contributed equally to this

work

${ }^{1}$ Institut de Systématique, Evolution et Biodiversité, UMR 7205 (CNRS,

MNHN, Sorbonne Université, EPHE), Muséum National d'Histoire Naturelle

CP50, 57 rue Cuvier, 75005 Paris, France

Full list of author information is available at the end of the article

(c) The Author(s) 2019. This article is distributed under the terms of the Creative Commons Attribution 4.0 International License (http://creativecommons.org/licenses/by/4.0/) which permits unrestricted use, distribution, and reproduction in any medium, provided you give appropriate credit to the original author(s) and the source, provide a link to the Creative Commons license, and indicate if changes were made. The Creative Commons Public Domain Dedication waiver (http://creativecommons.org/ publicdomain/zero/1.0/) applies to the data made available in this article, unless otherwise stated. 


\section{Background}

The evolution of complex phenotypes requiring the coordinated diversification of several traits is a puzzle. The persistence of intraspecific polymorphisms with several differentiated variants segregating within a single interbreeding population facilitates the identification of genes and epistatic interactions that control the developmental switches involved in their emergence. Unravelling the genetic architecture of polymorphic traits is therefore central to our understanding of the origins and evolutionary diversification of complex phenotypes.

The maintenance of discrete adaptive morphs that require the co-variation of multiple phenotypic modalities is surprising because interbreeding among morphs should lead to recombination between co-adapted loci, shuffling allelic combinations, and resulting in maladaptive intermediates. Recently, the dissection of several cases of balanced polymorphism has revealed a genetic architecture where a single Mendelian locus coordinates major phenotypic changes (see [1] for a review). These polymorphic loci could either be composed of a single causative gene with diverse pleiotropic effects, or of several tightly linked genes acting on one modality each, i.e. so-called "supergenes". Supergenes are usually described as clusters of two or more loci, each affecting a different morphological or behavioural trait; tight physical linkage and/or chromosomal inversions suppress recombination, such that multiple characters are inherited as a single Mendelian locus [2,3]. This architecture prevents suboptimal allelic combinations and results in the long-term co-existence of multiple well-differentiated variants (e.g. [4]), or conversely in reproductive isolation and speciation among ecotypes (e.g. [5]). In over 80 years since the proposal of this idea [6], supergenes have been reported in a wide range of complex polymorphisms, from adaptive colouration in butterflies and fish, through social structure in ants, to reproductive and behavioural strategies in animals and plants (e.g. [7-12]). The emergence of such genetic architecture, however, remains puzzling.

Despite the panoply of complex polymorphisms associated with supergenes, molecular evidence for the involvement of multiple genetic elements remains scarce. The individual genetic components have been characterised in detail only for the self-incompatibility supergenes in flowering plants $[13,14]$. For the polymorphism in wing colour pattern in Papilio polytes butterflies, controlled by a large inversion, supergene architecture was presumed for long time. However, recent gene expression data showed that this variation is controlled mostly by a single transcription factor doublesex, located within the inverted region and acting like a major developmental switch between different morphs via alternative splicing [15]. It remains unclear whether multiple genetic elements are functionally involved in this wing colour polymorphism. The concept of supergene is now a hot topic in biology, and identification of its functional elements (distinct genes vs. mutations in a single gene) is needed to shed light on the evolution of highly divergent polymorphic traits. Here, we apply different gene expression analyses to study the supergene that controls a well-documented polymorphism in a classical ecological model, the wing colour variation observed within populations of the neotropical butterfly $H$. numata.

Heliconius are chemically defended butterflies famous for their colourful wing patterns acting as warning signal towards predators [16]. Most species are involved in Müllerian mimicry associations with other locally abundant unpalatable species. For example, $H$. numata exhibits extraordinary resemblance to a number of species from the distantly related genus Melinaea, which diverged from the genus Heliconius over 90 million years ago [17]. A handful of genetic loci of major effect explain most of the variation in wing pattern within and among the majority of Heliconius species (reviewed in [18]). Recent studies have revealed the molecular identity the major loci: the transcription factor optix is responsible for turning on and off red, orange, and brown pattern elements [19], while the presumed cell cycle regulator cortex acts as a switch for black [20], and the regulatory gene aristaless controls white/yellow colours [21]. The morphogen WntA, in turn, controls the size and shape of the elements switched on and off by the first two loci [22]. These and a few other, yet unidentified loci belong to the genetic tool-kit that controls variation and produces both convergent and divergent wing colour patterns in Heliconius and other butterflies [23, 24].

In contrast to most Heliconius species where all individuals display the same colour pattern within each locality, $H$. numata shows a stable local polymorphism of wing colour pattern, with up to seven distinct morphs co-occurring in a single population [25]. This polymorphism is almost entirely determined by a single locus, the supergene $P$, found on linkage group 15 and characterised by long-range haplotypes in complete linkage disequilibrium [26]. Recombination in this region is suppressed due to the presence of two chromosomal inversions arranged in three distinct gene orders co-existing within populations [7] (Fig. 1). For example, the morph silvana is controlled by haplotype $P^{\text {sil }}$ which corresponds to the standard, ancestral gene arrangement, shared with most Heliconius species, while bicoloratus $\left(P^{b i c}\right)$ is determined by the first inversion, a 400-kb segment containing genes from Hnum000020 to Hnum000040; the second adjacent inversion, a 180-kb segment containing genes from Hnum000041 to Hnum000053, forms yet another gene order associated with several other morphs such 


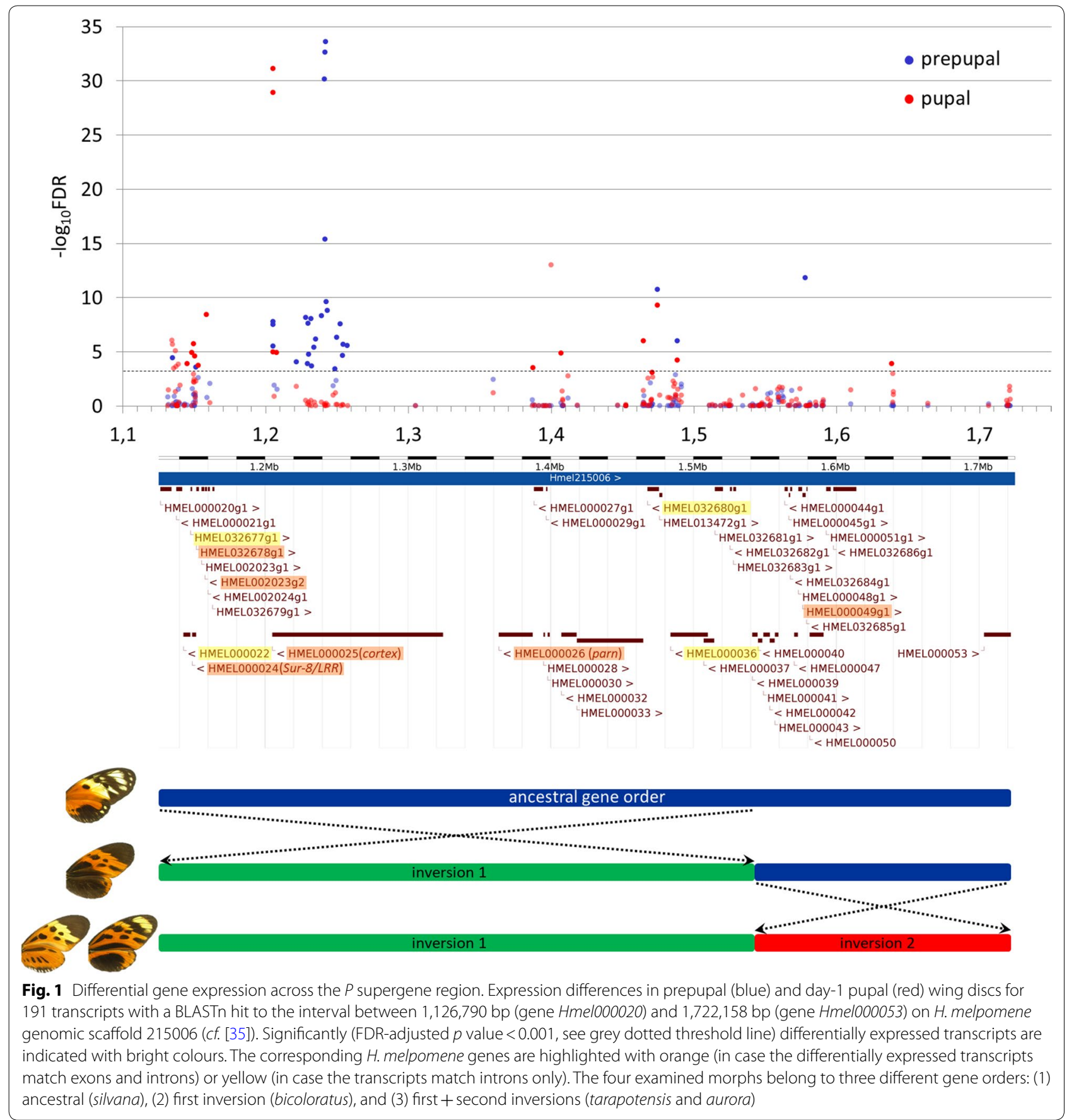

as tarapotensis ( $P^{\text {tar }}$ ) and aurora $\left(P^{a u r}\right)$. This supergene architecture presumably locks together the genetic combinations producing mimetic colour patterns: haplotypes controlling distinct morphs rarely recombine, therefore maintaining co-adapted alleles together. Remarkably, the $P$ locus contains the gene cortex, the expression of which in final instar larval wing discs is associated with some black elements on the hindwings of $H$. numata and
$H$. melpomene [19]. Is cortex the unique factor controlling the developmental switch between mimetic patterns in $H$. numata, similar to doublesex in $P$. polytes? Or are other genes within the $P$ locus involved in pattern variation as well, making the $P$ a classical supergene, i.e. "coadapted combination of several genes locked in inverted section of chromosome" [27]? Here, we apply different gene expression analyses to investigate the role of cortex 
and other genes within the $P$ supergene in controlling the variation of $H$. numata wing patterns. First, we perform RNA sequencing and differential expression analysis of the supergene $P$ genes at relevant stages of colour pattern development. We then investigate the differences in spatial expression patterns of candidate genes in the developing wing discs.

\section{Methods}

\section{Butterfly rearing}

Heliconius numata butterflies were collected in Tarapoto, Peru. Adults were fed with sugar water and pollen, and provided with Passiflora caerulea for egg deposition. Larvae were reared on $P$. caerulea and $P$. edulis plants.

\section{Genotyping assays}

The genotype of each individual at the supergene $P$ was determined using PCR-based genotyping assays based on intron length and SNP polymorphisms at the cortex gene (modified protocol of Chouteau et al. [28]). Genomic DNA was extracted from the bodies using Qiagen DNeasy Blood \& Tissue kit $c f$. the manufacturer's instructions. PCRs with forward 5'-CGTAGCGACCCG AGATTCTT and reverse 5'-ATACATGGCCACAGT TGATTC primers were carried out with $5 \mathrm{~min}$ at $94{ }^{\circ} \mathrm{C}$, followed by 35 cycles of $25 \mathrm{~s}$ at $94{ }^{\circ} \mathrm{C}, 25 \mathrm{~s}$ at $58{ }^{\circ} \mathrm{C}$, and $60 \mathrm{~s}$ at $72{ }^{\circ} \mathrm{C}$, and a final elongation phase at $72{ }^{\circ} \mathrm{C}$ for $5 \mathrm{~min}$. Gel electrophoresis was used to determine the size of the resulting PCR products: $374 \mathrm{bp}$ (present only in $P^{\text {sil }}$ haplotype), $925 \mathrm{bp}$ (present only in $P^{b i c}$ ), or $644 \mathrm{bp}$ (present in $P^{b i c}, P^{t a r}$, and $P^{a u r}$ ). The PCR products were also sequenced directly with the same primers to determine the genotype on the basis of diagnostic SNPs.

\section{Library preparation and RNA sequencing}

Individuals for RNA sequencing were collected from second generation of crosses between butterflies displaying aurora, bicoloratus, silvana, and tarapotensis phenotypes (Fig. 1). Wing discs were dissected in PBS from prepupae and day-1 pupae and stored in RNAlater. We used wing disc from prepupae with genotypes $P^{\text {aur }} / P^{\text {aur }}(n=4), P^{b i c}$, $P^{b i c}(n=4), P^{b i c} / P^{t a r}(n=3)$, and $P^{t a r} / P^{t a r}(n=3)$, and from day-1 pupae with genotypes $P^{\text {aur }} / P^{\text {aur }}(n=4), P^{\text {sil }} / P^{\text {sil }}$ $(n=3), P^{\text {tar }} / P^{\text {tar }}(n=3)$, resulting in 24 samples in total.

One forewing and one hindwing of each individual were homogenised in $350 \mu \mathrm{l}$ of RTL buffer with the Tissue Lyser (Qiagen, Hilden, Germany). Total RNA was extracted according to the manufacturer's protocol (RNeasy Mini kit, Qiagen, Hilden, Germany) and eluted in $30 \mu \mathrm{l}$ of RNase-free water. To avoid genomic contamination, RNase-free DNase treatment (Qiagen, Hilden, Germany) was performed during RNA extraction. RNA quality and concentration were measured with a Qubit
2.0 Fluorometer (Life Technologies) and a 2100 Bioanalyzer (Agilent Technologies).

Library preparation and Illumina sequencing were performed at the Ecole Normale Supérieure core genomic facility (Paris, France). Messenger (polyA+) RNAs were purified from $1 \mu \mathrm{g}$ of total RNA using oligo(dT). Libraries were prepared from pools of fore- and hindwing RNA of each individual using the TruSeq Stranded mRNA kit (Illumina). Twenty-four libraries (multiplexed by six on four flowcell lanes) were sequenced on Illumina HiSeq 1500 sequencer using 51 cycles per run, yielding $42 \pm 15$ million single 50-bp passing Illumina quality filter reads per sample. Sequencing lanes were randomized among samples.

\section{de novo transcriptome assembly and gene expression analyses}

All bioinformatics analyses were performed on the Galaxy server [29] of BioInformatics Platform for Agro-ecosystems Arthropods (BIPAA) of French National Institute for Agricultural Research (INRA) in Rennes, France. Low-quality $(<\mathrm{Q} 30)$ reads, adaptor sequences, and ribosomal RNA-like sequences were removed with Prinseq [30], Cutadapt [31], and riboPicker [32] tools, respectively. A total of $\sim 959,842,465$ high-quality clean reads from all samples were used to de novo assemble the wing disc transcriptome with the software Trinity [33] and following parameters: SS_lib_type $=F, k m e r \_s i z e=25$, max_pct_stdev $=100$, minimum contig length $=200 \mathrm{bp}$. Quality of the assembly was evaluated by estimating transcript abundance using the RSEM method [34] and subsequently computing N50 and ExN50 statistics. The latter takes into account the expression levels of each contig and is therefore a more suitable contig length metric for transcriptomes. To identify transcripts with orthologs in $H$. melpomene, all RNA sequences were aligned to the Hmel2_cds and Hmel2_scaffolds databases (downloaded from Lepbase v4 [35]) using NCBI BLASTn.

Reads from each sample were mapped to the assembled transcriptome using Bowtie2 [36]. Mapped reads for each transcript were counted with samtools idxstats [37]. Read counts were used to identify differentially expressed transcripts with the Bioconductor EdgeR package, as it performs best for datasets with small numbers of replicates [38].

Comparison of differential expression in genes located within the supergene with respect to the genes located in the rest of the genome were performed using gene set permutations test using the gene set enrichment analysis described in Subramanian et al. [39]. For each pair of phenotype within the two developmental stages, we used the 191 transcripts mapped to the supergene $P$ has a gene set and performed 1000 permutations to test whether the 
differential expression ranking significantly differed to the rest of the genome, using the GSEA software (http:// software.broadinstitute.org/gsea/index.jsp).

\section{Wing disc in situ hybridizations (ISH)}

Fragments ( 300 to $700 \mathrm{bp}$ long) of 28 candidate genes expressed within the $P$ interval (see Additional file 1: Table S1) were cloned into pCRII dual-promoter vector using the TA cloning kit (Life Technologies). Plasmids were isolated with QIAprep Spin Miniprep Kit (Qiagen) and used as template for PCR reactions with vector primers M13F and M13R. The amplified products were cleaned with QIAquick PCR purification kit (Qiagen) and used for SP6 or T7 transcription. Digoxigenin-labelled riboprobes were synthesised using SP6 and T7 RNA polymerases and DIG RNA labelling mix (Roche Applied Science).

Individuals used for ISH came from a $H$. numata stock (a mix of different morphs) maintained in the greenhouse at MNHN in Paris. At least five individuals of each (silvana, tarapotensis, and bicoloratus) morph were used for ISH. Wing discs were dissected in PBS from last instar larvae, fixed in $4 \%$ formaldehyde in PBS, gradually dehydrated and stored in methanol. ISH was performed $c f$. the protocol described previously in Martin and Reed [40]. Briefly, wing discs were gradually rehydrated, incubated $5 \mathrm{~min}$ with $25 \mu \mathrm{g} / \mathrm{ml}$ proteinase $\mathrm{K}$, post-fixed with $5.5 \%$ formaldehyde in PBS, and incubated in a standard hybridization buffer supplemented with $1 \mathrm{~g} / \mathrm{l}$ glycine and $30 \mathrm{ng} / \mathrm{ml}$ riboprobe for $20-24 \mathrm{~h}$ at $63{ }^{\circ} \mathrm{C}$. For secondary detection of the probe, wing discs were incubated in a 1:3000 dilution of anti-digoxigenin alkaline phosphatase Fab fragments and stained with BM Purple (Roche Applied Science) for 3-6 h at room temperature. Stained wing discs were photographed with a Leica DFC420 digital camera mounted on a Leica Z6 APO stereomicroscope.

\section{Results}

\section{Assembly of the H. numata wing disc transcriptome}

We performed a de novo transcriptome assembly using Illumina RNA sequencing data on fore- and hindwings of 24 individuals (14 prepupae and 10 day-1 pupae). Trinity yielded 53,719 transcripts with a cumulative length of $34.37 \mathrm{Mb}$ and the mean, median, and maximal transcript length of $640 \mathrm{bp}, 367 \mathrm{bp}$, and 33,692 bp, respectively. A large part of the assembled transcripts $(20,406$ out of 53,719$)$ fall within the size range of 201$300 \mathrm{bp} ; 9162$ transcripts are longer than $1000 \mathrm{bp}$, with 12 exceeding $10 \mathrm{~kb}$. We calculated the transcript N50 statistics of $980 \mathrm{bp}$. However, when excluding the transcripts expressed at very low levels, the maximal N50 value of $1356 \mathrm{bp}$ was found for $\mathrm{Ex}=88$, i.e. $50 \%$ of the total assembled nucleotides are included in the top $88 \%$ abundant transcripts $(N=7331)$ after normalising for read coverage. About $92.5 \%$ of the assembled sequences represent unique transcripts, whereas 4004 represent different isoforms of 2957 genes, some of which have up to 20 splicing variants. The transcriptome is available for download at Transcriptome Shotgun Assembly Sequence Database of NCBI (NCBI BioProject PRJNA555830).

A high-quality genome assembly is currently unavailable for $H$. numata. The assembly consists of more than 20,000 scaffolds with N50 of $61.3 \mathrm{~kb}$ ( $c f$. Lepbase v4 [35]). Therefore, we performed the analyses using the reference genome assembly Hmel2 (795 scaffolds, N50 $=2.1 \mathrm{Mb}$ [41]) of the closely related $H$. melpomene, diverged from $H$. numata around 4 Mya [42]. Of the 53,719 transcripts present in the $H$. numata wing disc transcriptome, more than $99 \%(53,301)$ could be aligned to the $H$. melpomene genomic scaffolds. However, only 27,999 H. numata transcripts were identified as orthologous to 12,835 out of 21,661 transcripts in H. melpomene coding DNA sequence (CDS) database. Such discrepancy might come from important alternative splicing variations between species, or incompleteness of $H$. melpomene CDS database.

We identified 191 transcripts corresponding to 169 unigenes (i.e. 157 transcripts with just one isoform and 12 transcripts with 2-8 isoforms) in the $H$. numata transcriptome that were aligned to the interval between 1,126,790 bp (gene Hmel000020) and 1,722,158 bp (gene Hmel000053) on H. melpomene genomic scaffold 215006, i.e. the interval homologous to the $P$ supergene. Of these 191 transcripts, 72 have a BLASTn hit to one of the 36 (out of the 41 present in this interval) predicted genes in the H. melpomene, with multiple short transcripts being part of the same gene. Hence, the majority of the genes in the $P$ supergene are expressed in the wing discs of $H$. numata at the prepupa and/or day-1 pupa stages, when wing pattern formation occurs. The genes not found in the transcriptome, and therefore not expressed at the relevant stages, are Hnum000051, Hnum002023g1, Hnum032679, Hnum032683, and Hnum032685. None of them is annotated for gene ontology terms.

\section{Differential expression within the $P$ supergene}

To identify the genes within the $P$ locus potentially associated with wing pattern development, we performed differential expression analysis using wing disc samples of different morphs (3-4 biological replicates per morph) at two developmental stages (prepupa and day-1 pupa), known to be important in wing pattern formation in other butterflies [43]. Principal component analysis showed a clear segregation of prepupal and day-1 
pupa samples (circles vs. squares in Additional file 1: Figure S1), with a single tarapotensis pupa as an outlier. Overall, 1048 (in prepupa) and 3488 (in day-1 pupa) of the 53,719 transcripts were identified as differentially expressed between morphs using a false discovery rate (FDR) adjusted $p$ value of $<0.001$ and at least a twofold change in the expression level. Neither WntA nor optix, two major wing patterning genes in Lepidoptera (acting at larval and late pupal stages, respectively $[19,22])$, and unlinked to the $P$ interval, show differences in expression levels among $H$. numata morphs. The differential expression within the supergene was generally more marked than in the rest of the transcriptome, as revealed by gene set enrichment analysis (see Additional file 1: Table S2). Within the $P$ interval, 30 and 24 of the transcripts mapping to the supergene were indeed differentially expressed among morphs at the prepupa and day- 1 pupa stages, respectively (bright blue and red dots in Fig. 1).

However, seven of the 24 transcripts had very high expression levels in a single tarapotensis day-1 pupa (an outlier in the PCA plot, see Additional file 1: Figure S1) in comparison with two other samples of the same morph and therefore were not taken into consideration in further analyses (pale red dots above the threshold line in Fig. 1). Moreover, the majority of differentially expressed transcripts within the $P$ interval generally have low expression levels as indicated by negative $\log \mathrm{CPM}$ values (Table 1).

Five transcripts were expressed differentially at both developmental stages (Table 1). Three of them corresponded to the gene cortex, known to play a role in wing pattern formation in Heliconius and other Lepidoptera $[20,44]$, whereas the other two matched the introns of Hmel032680 (predicted WD repeat-containing protein) and Hmel000036 (predicted WAS protein homologue 1). Moreover, 22 transcripts expressed differentially in prepupae corresponded to the very large (more than $100 \mathrm{~kb}$ ) first intron of cortex. At this stage, expression of cortex CDS and the majority of intron transcripts was highest in the bicoloratus homozygotes $\left(P^{b i c} / P^{b i c}\right)$, intermediate in bicoloratus heterozygotes ( $\left.P^{b i c} / P^{\text {tar }}\right)$ and aurora, and lowest in tarapotensis. In day-1 pupae, expression of cortex CDS was highest in silvana, intermediate in aurora, and lowest in tarapotensis. However, only one transcript that corresponded to the first intron of cortex was differentially expressed at this stage. To establish whether the transcripts in the first intron of cortex represent alternative splicing variants (as has been observed in H. melpomene [20]), we performed BLASTn to $H$. numata scaffolds on Lepbase v4 [35] and examined 20-bp sequences flanking those transcripts for presence of GT-AG, indicative of intron splicing sites (see Additional file 1: Table S3). At least nine transcripts have both
AG and GT in the flanking $5^{\prime}$ and $3^{\prime}$ regions, respectively, suggesting that those could be alternative splicing forms of cortex, whereas other transcripts could be distinct non-coding RNAs.

Furthermore, two transcripts corresponding to different parts of Hnum000024 (predicted similar to Sur-8, a positive regulator of Ras signalling) CDS were expressed differentially in both prepupae (comp35224_c0_seq1, high in homozygote bicoloratus and tarapotensis, low in aurora) and day-1 pupae (comp35900_c0_seq2, high in tarapotensis, lowest in silvana), see Table 1 . We also found Hnum000049 (predicted protein coding) to be upregulated in bicoloratus homo- and heterozygotes at the prepupa stage. Gene Hnum000026 (putative poly(A)specific ribonuclease) was upregulated in tarapotensis and downregulated in silvana (in comparison with aurora) in day-1 pupae, whereas Hnum032678 (predicted protein coding) showed the opposite pattern. Finally, gene Hnum002023g2 (predicted protein coding) was downregulated in both silvana and tarapotensis at that stage.

\section{Differential expression in relation to supergene structure}

Our experimental design allows for comparison of gene expression across different gene arrangements within the $P$ supergene (Fig. 1). For instance, day-1 pupa stage allows for comparison of the ancestral gene arrangement in silvana versus both first $(400-\mathrm{kb}$ segment containing genes Hnum000020-Hnum000040) and second (180-kb segment containing genes Hnum000041-Hnum000053) inversions characteristic of tarapotensis and aurora. We found eight transcripts (indicated with * in Table 1) to be consistently up- or downregulated in silvana and showing the opposite pattern of expression in tarapotensis and aurora. Remarkably, the first four of those are located near the left breakpoint, whereas the other four are closer to the right breakpoint of the first inversion. Some of these transcripts correspond to introns of predicted genes or intergenic sequences. Hence, the first inversion does not only affect expression levels of the existing genes, but also introduces novel expression patterns. Morphs analysed at the prepupa stage, on the other hand, differ in the presence (in tarapotensis and aurora) or absence (in bicoloratus) of the second inversion. The presence of this genomic rearrangement is associated with decreased expression of transcript comp166797_c0_ seq1 (Hnum000049).

Variation in cortex expression patterns in larval wing discs To establish the relationships between expression and adult wing patterns, we performed in situ hybridization (ISH) with probes against 28 candidate genes (see Additional file 1: Table S1) from the $P$ supergene. As 


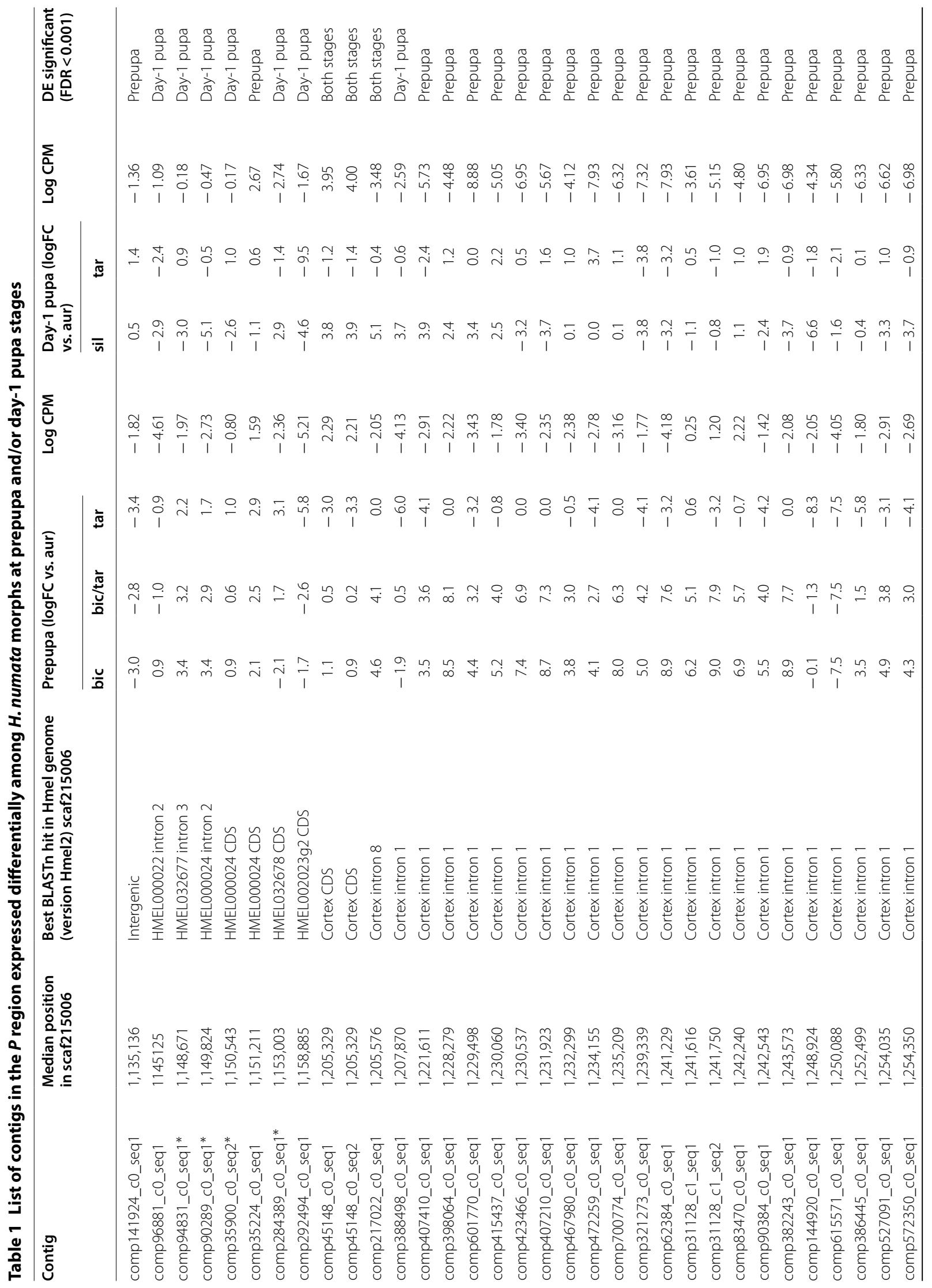




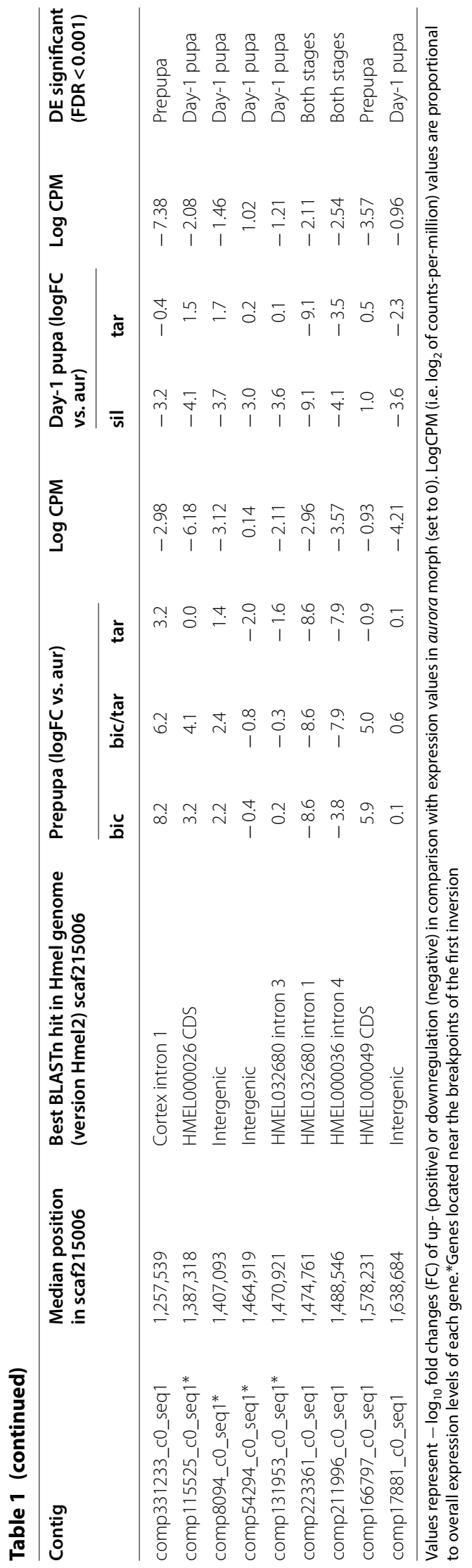


the prepupal and day-1 pupal wings are too fragile to be used in ISH, we performed the latter on the wing discs from the last instar larvae. For all but one candidate, ISH resulted either in the ubiquitous expression or in the absence of any detectable signal (see Additional file 1: Figure S2 for examples). However, in line with the previous study [20], clear differences in the spatial patterns of expression were observed for the cortex gene (Fig. 2 and Additional file 1: Figure S3). In particular, the pattern of cortex signal in larval hindwings reflects the amount of black pigment in the adults. Moreover, cortex is expressed in the black forewing tip in bicoloratus (but not in other morphs), and in the so-called "comma mark" on the forewing of silvana. These results are consistent with the findings of the differential expression analyses, e.g. high expression levels of cortex in bicoloratus and low in tarapotensis at the prepupa stage.

\section{Discussion}

In addition to developing the first transcriptome resource for $H$. numata, we performed differential expression analyses at two developmental stages to identify genes involved in variation of wing colour patterns in this species. A large number of genes throughout the transcriptome were differentially expressed among $H$. numata morphs: 1048 (out of 53,719) at prepupa and 3488 at day-1 pupa stage, providing a long list of putative candidates. However, because variation in $H$. numata colour pattern is known to be controlled by a single Mendelian locus located in the $P$ region [26], these transcriptional differences could be explained by (a) downstream responses to signals coming from the supergene and cosegregating parts of the genome and/or (b) physiological response to fluctuating field conditions in Peru. This highlights the need for mapping the loci of interest prior to differential expression analyses in order to limit the number of candidate genes identified by RNA sequencing, and target the causative region.

By specifically focusing on the $P$ locus, previously shown to control colour pattern variation in $H$. numata [7], our experiments, even though they were limited by a number of morphs, replicates, and developmental time points, confirm the role of cortex in the variation of some black pattern elements on the wings across $H$. numata morphs. Colour pattern phenotypes caused by cortex expression in $H$. melpomene and $H$. numata strikingly differ, suggesting that the emergence of novel patterns is caused by heterotopic expression, i.e. changes in the spatial regulation of cortex. Similar to optix which causes variation in red pattern elements [45], the evolution of black colour patterns within and among Heliconius species may be mainly driven by evolution of regulatory regions, changing the localisation of gene expression throughout the wing which is, in turn, determined by upstream positional signals. As in $H$. melpomene, differential expression was not confined to cortex exons [20], as 22 transcripts up- or downregulated at the prepupa stage map to its first intron and could indicate unidentified splice variants or non-coding RNAs.

We also found that the majority of genes in the $P$ interval were expressed in the developing wing discs during the critical stages of colour pattern formation, and nine of them were expressed differentially among morphs. In addition, expression levels of several contigs corresponding to intronic and intergenic regions correlate with the presence/absence of the chromosomal inversions within the $P$ interval. Large genomic rearrangements are frequently associated with gene silencing or ectopic expression patterns (e.g. [46, 47]). Additional studies of gene expression and function will investigate whether these changes indeed play a role in colour pattern variation in H. numata.

Most of the genes within the $P$ interval are expressed in (pre)pupal wing discs, and quantitative as well as qualitative variation in expression of multiple genes has been detected between $P$ genotypes, suggesting that inversions are associated with drastic changes in expression throughout the $P$ region. Furthermore, variation in cortex expression revealed by ISH is restricted to certain parts of the wing and therefore does not fully recapitulate the variation in adult wing pattern, suggesting that other genes located in the $P$ interval may play a role in colour pattern variations, independently of variation in black elements observed across $H$. numata morphs. The technical limit of ISH focusing on the restricted developmental windows may prevent us to detect other genes involved in the developmental switch between mimetic patterns in $H$. numata. Identifying the functional role of cortex using CRISPR/Cas9 knockout, for instance, would help understanding its implication in the development of different colour patterns in $H$. numata and its putative interactions with other genes.

Nevertheless, our current results are overall consistent with the hypothesis that the $P$ locus acts as a classical supergene, whereby several linked genes are involved in the developmental switch between the different mimetic patterns. It should be noted that the second inversion has been recently shown to be followed by a third rearrangement, whose limits are not fully determined yet (unp. data), so that genes located further away from the region studied here could also be involved in the developmental switch.

Even though our ISH experiments performed at late larval stage cannot fully demonstrate the supergene hypothesis, our study opens up new perspectives on the architecture of the $P$ locus in $H$. numata. Because of the 


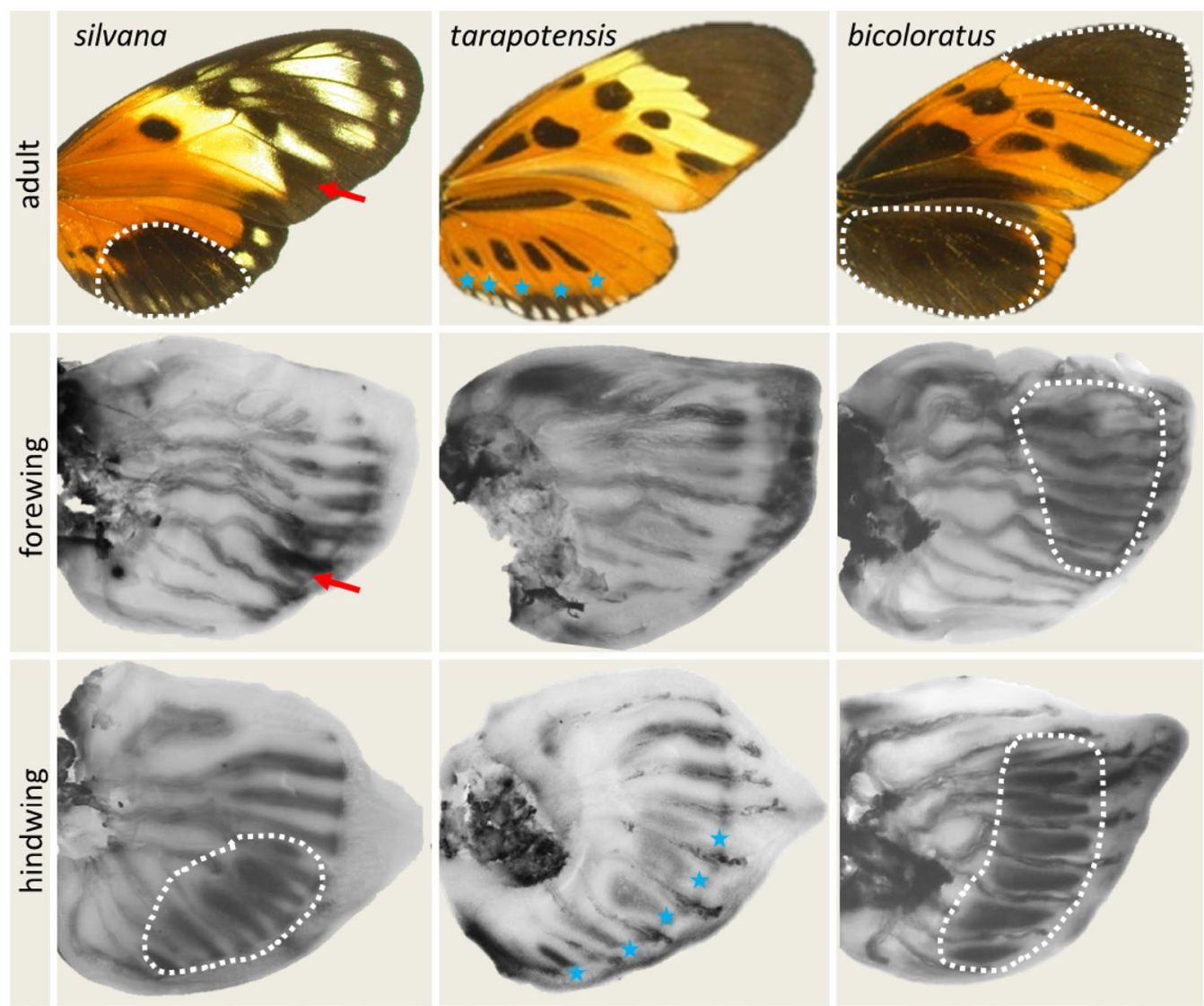

Fig. 2 Expression patterns of cortex in larval wing discs of different H. numata morphs. Expression of cortex in late larval wing discs is associated with the amount of black pigment in the hindwings (top and bottom panels, stars and dashed lines indicate corresponding areas of the wings), the "comma mark" on silvana forewing (red arrows), and the black forewing tip in bicoloratus

technical constraints of ISH on wing disc, we studied only a limited time window of wing pattern formation. However, variation in colour patterns could be triggered by heterochrony in gene expression, or differential expression at stages not targeted here. Further studies on extended developmental time series are required to uncover full genetic control of such striking variation and to investigate the genetic architecture of the $P$ supergene.

\section{Conclusions}

Here, we developed the first transcriptome resource for $H$. numata and performed differential expression analyses during wing development to identify genes involved in forming the supergene and contributing to the expression of differentiated phenotypes. Our analyses confirm the role of cortex in the formation of black wing pattern elements. Our results are consistent with the hypothesis that other genes in the $P$ region may play a role in colour variation in $H$. numata. Functional studies on the gene cortex and exploration of longer developmental time series will now be required to conclude whether the supergene $P$ is a classical supergene, or whether cortex alone fully controls the developmental switches involved in colour pattern polymorphism in H. numata.

\section{Additional files}

Additional file 1: Table S1. Information on probes used for ISH in $\mathrm{H}$. numata larval wing discs. Figure S1. Principal component analysis (PCA) plot of read counts matrix from RNA-seq data. Table S2. Gene set enrichment analysis comparing the rank of differential expression in transcripts mapped to the supergene $P$ to the rest of the transcriptome using 1,000 transcript permutations. Table S3. Analysis of splicing sites in the first intron of cortex. Figure S2. Examples of expression patterns observed in larval wing discs of H. numata. A. Ubiquitous expression, B. Expression in the trachea, C. No detectable signal. Figure S3. Expression patterns of cortex in larval wing discs of $H$. numata (all samples).

\section{Acknowledgements}

We thank Monica Arias and Nicolas Chazot for help with butterfly rearing, and Anthony Bretaudeau and Fabrice Legeai from BIPAA for help with RNA-seq analyses. 


\section{Authors' contributions}

SVS performed RNA analyses and ISH, and wrote the manuscript. MC collected and reared butterflies, designed and performed the controlled crosses. FPP performed the RNA extractions and GSEA analyses. CB performed library preparation and RNA sequencing. $\mathrm{VL}$ and $\mathrm{MJ}$ conceived and supervised the whole study. All authors read and approved the final manuscript.

\section{Funding}

This work was funded by a young research grant from the French National Agency for Research (ANR-13-JSV7-0003-01-Domevol) and an Emergence grant from Mairie de Paris obtained to VL. Sequencing was supported by the France Génomique national infrastructure, funded as part of the "Investissements d'Avenir" programme managed by the Agence Nationale de la Recherche (ANR-10-INBS-09). MJ was funded by ANR grants ANR-12-JSV7-0005 and ANR-18-CE02-0019-01.

\section{Availability of data and materials}

The datasets generated and analysed during the current study are available in the NCBI SRA and TSA repositories (PRJNA555830).

\section{Ethics approval and consent to participate}

The animals used in this study do not require ethics approval or consent to participate.

\section{Consent for publication}

Not applicable.

\section{Competing interests}

The authors declare that they have no competing interests.

\begin{abstract}
Author details
${ }^{1}$ Institut de Systématique, Evolution et Biodiversité, UMR 7205 (CNRS, MNHN, Sorbonne Université, EPHE), Muséum National d'Histoire Naturelle CP50, 57 rue Cuvier, 75005 Paris, France. ${ }^{2}$ Laboratoire Ecologie, Evolution, Interactions Des Systèmes Amazoniens (LEEISA), USR 3456, CNRS Guyane, Université De Guyane, 275 route de Montabo, 97334 Cayenne, French Guiana. ${ }^{3}$ Genomic Facility, Institut de Biologie de I'Ecole normale superieure (IBENS), École normale supérieure, CNRS, INSERM, PSL Université Paris, 75005 Paris, France. ${ }^{4}$ Centre d'Ecologie Fonctionnelle et Evolutive, UMR 5175 CNRS-Université de Montpellier, École Pratique des Hautes Études, Université Paul Valéry, 34293 Montpellier 5, France.
\end{abstract}

Received: 28 February 2019 Accepted: 12 July 2019

Published online: 08 August 2019

\section{References}

1. Llaurens V, Whibley A, Joron M. Genetic architecture and balancing selection: the life and death of differentiated variants. Mol Ecol. 2017;26(9):2430-48.

2. Schwander T, Libbrecht R, Keller L. Supergenes and complex phenotypes. Curr Biol. 2014;24(7):R288-94.

3. Thompson MJ, Jiggins CD. Supergenes and their role in evolution. Heredity. 2014;113(1):1-8.

4. Twyford AD, Friedman J. Adaptive divergence in the monkey flower Mimulus guttatus is maintained by a chromosomal inversion. Evolution. 2015;69(6):1476-86.

5. Hermann K, Klahre U, Moser M, Sheehan H, Mandel T, Kuhlemeier C. Tight genetic linkage of prezygotic barrier loci creates a multifunctional speciation island in Petunia. Curr Biol. 2013;23(10):873-7.

6. Fisher RA. The genetical theory of natural selection. Oxford: Oxford University Press; 1930.

7. Joron M, Frezal L, Jones RT, Chamberlain NL, Lee SF, Haag CR, et al. Chromosomal rearrangements maintain a polymorphic supergene controlling butterfly mimicry. Nature. 2011;477(7363):203-6.

8. Lawson LP, Vander Meer RK, Shoemaker D. Male reproductive fitness and queen polyandry are linked to variation in the supergene Gp-9 in the fire ant Solenopsis invicta. Proc Biol Sci. 2012;279(1741):3217-22.

9. Brennan AC. Distyly supergenes as a model to understand the evolution of genetic architecture. Am J Bot. 2017;104(1):5-7.
10. Küpper C, Stocks M, Risse JE, Dos Remedios N, Farrell LL, McRae SB, et al. A supergene determines highly divergent male reproductive morphs in the ruff. Nat Genet. 2016;48(1):79-83.

11. Tuttle EM, Bergland AO, Korody ML, Brewer MS, Newhouse DJ, Minx P, et al. Divergence and functional degradation of a sex chromosome-like supergene. Curr Biol. 2016;26(3):344-50.

12. Kirubakaran TG, Grove H, Kent MP, Sandve SR, Baranski M, Nome T, et al. Two adjacent inversions maintain genomic differentiation between migratory and stationary ecotypes of Atlantic cod. Mol Ecol. 2016;25(10):2130-43.

13. Wheeler MJ, de Graaf BH, Hadjiosif N, Perry RM, Poulter NS, Osman K, et al. Identification of the pollen self-incompatibility determinant in Papaver rhoeas. Nature. 2009;459(7249):992-5.

14. Li J, Cocker JM, Wright J, Webster MA, McMullan M, Dyer S, et al. Genetic architecture and evolution of the $S$ locus supergene in Primula vulgaris. Nat Plants. 2016;2(12):16188.

15. Kunte K, Zhang W, Tenger-Trolander A, Palmer DH, Martin A, Reed RD, et al. Doublesex is a mimicry supergene. Nature. 2014;507(7491):229-32.

16. Merrill RM, Dasmahapatra KK, Davey JW, Dell'Aglio DD, Hanly JJ, Huber B, et al. The diversification of Heliconius butterflies: what have we learned in 150 years? J Evol Biol. 2015;28(8):1417-38.

17. Wahlberg N, Leneveu J, Kodandaramaiah U, Peña C, Nylin S, Freitas AV, et al. Nymphalid butterflies diversify following near demise at the Cretaceous/Tertiary boundary. Proc Biol Sci. 2009;276(1677):4295-302.

18. Nadeau NJ. Genes controlling mimetic colour pattern variation in butterflies. Curr Opin Insect Sci. 2016;17:24-31.

19. Reed RD, Papa R, Martin A, Hines HM, Counterman BA, Pardo-Diaz C, et al. Optix drives the repeated convergent evolution of butterfly wing pattern mimicry. Science. 2011;333(6046):1137-41.

20. Nadeau NJ, Pardo-Diaz C, Whibley A, Supple MA, Saenko SV, Wallbank RW, et al. The gene cortex controls mimicry and crypsis in butterflies and moths. Nature. 2016;534(7605):106-10.

21. Westerman EL, VanKuren NW, Massardo D, Tenger-Trolander A, Zhang W, Hill Rl, et al. Aristaless controls butterfly wing color variation used in mimicry and mate choice. Curr Biol. 2018;28(21):3469-74.

22. Martin A, Papa R, Nadeau NJ, Hill Rl, Counterman BA, Halder G, Jiggins CD, et al. Diversification of complex butterfly wing patterns by repeated regulatory evolution of a Wnt ligand. Proc Natl Acad Sci USA. 2012;109(31):12632-7.

23. Mazo-Vargas A, Concha C, Livraghi L, Massardo D, Wallbank RWR, Zhang $L$, et al. Macroevolutionary shifts of WntA function potentiate butterfly wing-pattern diversity. Proc Natl Acad Sci USA. 2017:114(40):10701-6.

24. Zhang L, Mazo-Vargas A, Reed RD. Single master regulatory gene coordinates the evolution and development of butterfly color and iridescence. Proc Natl Acad Sci USA. 2017;114(40):10707-12.

25. Joron M, Wynne IR, Lamas G, Mallet J. Variable selection and the coexistence of multiple mimetic forms of the butterfly Heliconius numata. Evol Ecol. 1999;13:721-54.

26. Joron M, Papa R, Beltrán M, Chamberlain N, Mavárez J, Baxter S, et al. A conserved supergene locus controls colour pattern diversity in Heliconius butterflies. PLoS Biol. 2006;4(10):e303.

27. Dobzhansky T. Genetics of the evolutionary process. New York: Colombia University Press; 1970.

28. Chouteau M, Llaurens V, Piron-Prunier F, Joron M. Polymorphism at a mimicry supergene maintained by opposing frequency-dependent selection pressures. Proc Natl Acad Sci USA. 2017;114(31):8325-9.

29. Afgan E, Baker D, Batut B, van den Beek M, Bouvier D, Čech M, et al. The Galaxy platform for accessible, reproducible and collaborative biomedical analyses: 2018 update. Nucl Acids Res. 2018;46(W1):W537-44.

30. Schmieder R, Edwards R. Quality control and preprocessing of metagenomic datasets. Bioinformatics. 2011;27:863-4.

31. Martin M. Cutadapt removes adapter sequences from high-throughput sequencing reads. EMBnet J. 2011;17(1):10-2.

32. Schmieder R, Lim YW, Edwards R. Identification and removal of ribosomal RNA sequences from metatranscriptomes. Bioinformatics. 2012:28(3):433-5.

33. Grabherr MG, Haas BJ, Yassour M, Levin JZ, Thompson DA, Amit I, et al. Full-length transcriptome assembly from RNA-Seq data without a reference genome. Nat Biotechnol. 2011;29(7):644-52.

34. Li B, Dewey CN. RSEM: accurate transcript quantification from RNA-Seq data with or without a reference genome. BMC Bioinform. 2011;12:323. 
35. Challis RJ, Kumar S, Dasmahapatra KK, Jiggins CD, Blaxter M. Lepbase: the Lepidopteran genome database. bioRxiv. 2016;1:1. https://doi. org/10.1101/056994.

36. Langmead B, Salzberg S. Fast gapped-read alignment with Bowtie 2. Nat Methods. 2012;9:357-9.

37. Li H, Handsaker B, Wysoker A, Fennell T, Ruan J, Homer N, et al. 1000 Genome project data processing subgroup. The sequence alignment/ map format and SAMtools. Bioinformatics. 2009;25(16):2078-9.

38. Schurch NJ, Schofield P, Gierliński M, Cole C, Sherstnev A, Singh V, et al. How many biological replicates are needed in an RNA-seq experiment and which differential expression tool should you use? RNA. 2016;22(6):839-51.

39. Subramanian A, Tamayo P, Mootha VK, Mukherjee S, Ebert BL, Gillette MA, Paulovich A, Pomeroy SL, Golub TR, Lander ES. Gene set enrichment analysis: a knowledge-based approach for interpreting genome-wide expression profiles. PNAS. 2005;102(43):15545-50.

40. Martin A, Reed RD. Wnt signaling underlies evolution and development of the butterfly wing pattern symmetry systems. Dev Biol. 2014;395(2):367-78.

41. Davey JW, Chouteau M, Barker SL, Maroja L, Baxter SW, Simpson F, et al. Major improvements to the Heliconius melpomene genome assembly used to confirm 10 chromosome fusion events in 6 million years of butterfly evolution. G3 (Bethesda). 2016;6(3):695-708.
42. Kozak KM, Wahlberg N, Neild AF, Dasmahapatra KK, Mallet J, Jiggins CD. Multilocus species trees show the recent adaptive radiation of the mimetic heliconius butterflies. Syst Biol. 2015;64(3):505-24.

43. Beldade P, Peralta CM. Developmental and evolutionary mechanisms shaping butterfly eyespots. Curr Opin Insect Sci. 2017;19:22-9.

44. Van't Hof AE, Campagne P, Rigden DJ, Yung CJ, Lingley J, Quail MA, et al. The industrial melanism mutation in British peppered moths is a transposable element. Nature. 2016;534(7605):102-5.

45. Wallbank RW, Baxter SW, Pardo-Diaz C, Hanly JJ, Martin SH, Mallet J, et al, Evolutionary novelty in a butterfly wing pattern through enhancer shuffling. PLoS Biol. 2016;14(1):e1002353.

46. Hough RB, Lengeling A, Bedian V, Lo C, Bućan M. Rump white inversion in the mouse disrupts dipeptidyl aminopeptidase-like protein 6 and causes dysregulation of Kit expression. Proc Natl Acad Sci USA. 1998;95(23):13800-5.

47. Puig M, Cáceres $M$, Ruiz A. Silencing of a gene adjacent to the breakpoint of a widespread Drosophila inversion by a transposon-induced antisense RNA. Proc Natl Acad Sci USA. 2004;101(24):9013-8.

\section{Publisher's Note}

Springer Nature remains neutral with regard to jurisdictional claims in published maps and institutional affiliations.
Ready to submit your research? Choose BMC and benefit from:

- fast, convenient online submission

- thorough peer review by experienced researchers in your field

- rapid publication on acceptance

- support for research data, including large and complex data types

- gold Open Access which fosters wider collaboration and increased citations

- maximum visibility for your research: over $100 \mathrm{M}$ website views per year

At BMC, research is always in progress.

Learn more biomedcentral.com/submissions 